\title{
Dependence on Frequency of the Electromagnetic Field Distribution inside a Cylindrical Cavity Excited through an Off-Axis Aperture
}

\author{
Fabrizio Consoli, ${ }^{1}$ Luigi Celona, ${ }^{1}$ Giovanni Ciavola, ${ }^{1}$ Santo Gammino, ${ }^{1}$ Fabio Maimone, ${ }^{1}$ \\ Rocco Salvatore Catalano, ${ }^{2}$ Sebastiano Barbarino, ${ }^{2,3}$ David Mascali, $^{3}$ and Livia Tumino ${ }^{2}$ \\ ${ }^{1}$ Laboratori Nazionali del Sud, Istituto Nazionale di Fisica Nucleare, Via S. Sofia 62, 95123 Catania, Italy \\ ${ }^{2}$ Dipartimento di Fisica e Astronomia, Università degli Studi di Catania, Via S. Sofia 64, 95123 Catania, Italy \\ ${ }^{3}$ Dipartimento di Ingegneria Informatica e delle Telecomunicazioni, Università degli Studi di Catania, \\ Viale A. Doria 6, 95125 Catania, Italy \\ Correspondence should be addressed to Fabrizio Consoli, consoli@lns.infn.it \\ Received 18 November 2007; Accepted 18 March 2008 \\ Recommended by Tibor Berceli
}

To explain the relevant changes in the electron cyclotron resonance ion source behaviour for small variations of the exciting radiation frequency, we determine the spatial distribution of the field within the cavity for every resonant mode.

Copyright (c) 2008 Fabrizio Consoli et al. This is an open access article distributed under the Creative Commons Attribution License, which permits unrestricted use, distribution, and reproduction in any medium, provided the original work is properly cited.

\section{INTRODUCTION}

In we want to study the electromagnetic field distribution in a perfectly conductive cylindrical cavity, for every resonant mode excited by a rectangular waveguide operating in the microwave range, through an aperture placed off axis on the top circular base (see Figure 1).

This situation is actually met in the modern electron cyclotron resonance (ECR) ion sources, where plasma is magnetically confined and excited by microwave fields. It was experimentally observed [1] that the plasma formation, the consequent amount of particles extracted from the source, and the related beam shape strongly depend on the frequency of the electromagnetic wave feeding the cavity. Indeed, by considering an ideal cavity, the frequency variation of the incoming radiation causes the excitation of a discrete number of modes (see Figure 1(b)), each coupled differently with the off-axis waveguide. Then, the electromagnetic field distribution inside the cavity will be different for each resonant frequency. Therefore, the particle motion will be affected by the selected mode, that is, by the excitation frequency. On the purpose to give a quantitative explanation of the problem we propose, in the preliminary phase of this work, to give the description of the electromagnetic field within the cavity following Van Bladel's approach [2]. We consider as a reference for this study the experimental setup represented by the SERSE ion source operating at INFN-LNS in Catania since 1998 [3, 4], but the analysis described in the following is applicable to any similar apparatus.

\section{DESCRIPTION OF THE EXPERIMENTAL SETUP}

The particles inside the ion source cavity are subjected to a nonuniform confining magnetostatic field $\vec{B}$. It is possible to consider it as generated by the superimposition of a hexapole and two solenoids:

$$
\begin{aligned}
& B_{x}=x\left(-B_{1} z+2 S_{\mathrm{ex}} y\right), \\
& B_{y}=-B_{1} y z+S_{\mathrm{ex}}\left(x^{2}-y^{2}\right), \\
& B_{z}=B_{0}+B_{1} z^{2}
\end{aligned}
$$

where $S_{\text {ex }}$ is a constant related to the hexapole field, $B_{0}$ and $B_{1}$ to the solenoids ones. For the sake of simplicity, we consider 


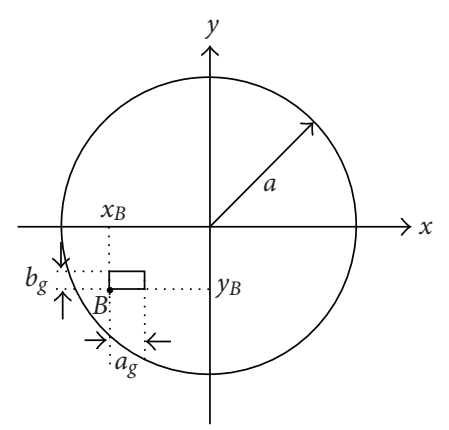

(a)

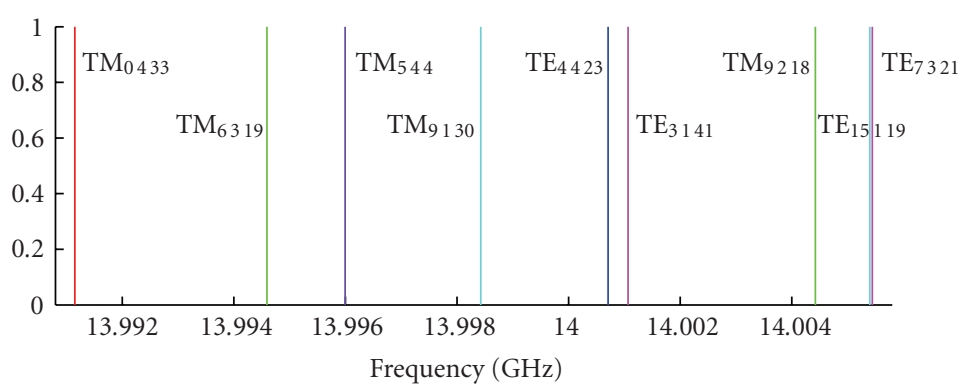

(b)

FIgURE 1: Scheme of the top base of the cylindrical cavity with the feeding waveguide, $a=6.5 \mathrm{~cm}, L$ (cavity length) $=45 \mathrm{~cm}, a_{g}=2 b_{g}=$ $15.8 \mathrm{~mm}, x_{B}=-40.4 \mathrm{~mm}, y_{B}=-28.95 \mathrm{~mm}$. (b) Mode distribution near to $14 \mathrm{GHz}$, usual source excitation frequency.

here the presence of only one electron inside the cavity. The equation describing its motion in a magnetostatic field is

$$
\frac{d \vec{v}}{d t}=\frac{q}{m_{0} \gamma(v)}[\vec{v} \times \vec{B}],
$$

where $\vec{v}, q$, and $m_{0}$ are the velocity, the charge, and the rest mass of the electron, $\gamma(\nu)=\left[1-(\nu / c)^{2}\right]^{-0.5}$ is the relativistic factor, $c$ is the light velocity in vacuum, and $\vec{B}=B_{z} \hat{x}+B_{y} \hat{y}+B_{z} \hat{z}$ is given by the formulas (1). The particle trajectory achieved by the numerical solution of this differential equation with given initial conditions has a projection in the $x y$ plane for $z=L / 2$, like that star shape represented in all the pictures of Figure 2. By considering the different patterns achieved by varying these initial conditions, it was possible to observe that the motion shape and the directions of the trajectories tips are quite similar one to the others because they are determined by the magnetostatic field only.

\section{DISTRIBUTION OF THE ELECTROMAGNETIC FIELD WITHIN THE CAVITY}

In Figure 1(a), the scheme of the cylindrical cavity is shown with its feeding WR62 rectangular waveguide placed far from the cavity axis. The fields in stationary conditions, inside a lossless cavity in vacuum can be written as [2]

$$
\begin{aligned}
\vec{E}= & -\sum_{m}\left[c \frac{\omega_{m}}{\omega_{m}^{2}-\omega^{2}} \frac{\int_{S}\left(\hat{z} \times \vec{E}_{\mathrm{TE} 10}\right) \cdot \vec{h}_{m} d S}{\int_{V}\left|\vec{e}_{m}\right|^{2} d V}\right] \vec{e}_{m}, \\
\vec{H}= & -\frac{1}{i \omega \mu} \sum_{m}\left[\frac{\int_{S}\left(\hat{z} \times \vec{E}_{\mathrm{TE} 10}\right) \cdot \vec{g}_{m} d S}{\int_{V}\left|\vec{g}_{m}\right|^{2} d V}\right] \vec{g}_{m} \\
& +\sum_{m}\left[\frac{1}{i \mu} \frac{\omega}{\omega_{m}^{2}-\omega^{2}} \frac{\int_{S}\left(\hat{z} \times \vec{E}_{\mathrm{TE} 10}\right) \cdot \vec{h}_{m} d S}{\int_{V}\left|\vec{h}_{m}\right|^{2} d V}\right] \vec{h}_{m},
\end{aligned}
$$

where the index $m$ stands for a triple set of indices, $\mu=$ $4 \pi 10^{-7} \mathrm{H} / \mathrm{m}, c=299792458 \mathrm{~m} / \mathrm{s}, S$ is the excitation aperture surface, $V$ is the cavity volume, $\omega$ and $\omega_{m}$ are the excitation and the mode characteristic angular frequencies, $\vec{h}_{m}$ and $\vec{e}_{m}$ are the solenoidal magnetic and electric eigenvectors (with $\left.\vec{h}_{m}=\left(c / \omega_{m}\right) \vec{\nabla} \times \vec{e}_{m}\right), \vec{g}_{m}$ is the irrotational magnetic eigenvector, and

$$
\vec{E}_{\mathrm{TE} 10}=-A i e^{i \omega t} \frac{\omega \mu a_{g}}{\pi} \sin \left(\frac{\pi x}{a_{g}}\right) \hat{y}
$$

is the field of the dominant mode in the rectangular waveguide. We indicate with $a_{g}$ the waveguide width (see Figure 1), and $A$ is a constant related to the waveguide power. If the frequency of the incoming wave does not coincide with one of the $\omega_{m}$ in (3), the field in the ideal lossless cavity is the sum of different finite terms, contributing to the total energy in the cavity. If, otherwise, for a given $\bar{m}$ the frequency $\omega$ coincides exactly with $\omega_{\bar{m}}$, the term in (3) relative to this mode will diverge, that is, it will become much larger than the others. In the time domain, it means that the energy in the cavity constantly increases with time and with a rate depending on the coupling between the incoming wave and the mode $\bar{m}[5]$. An accurate general representation of the fields at the resonance can be therefore obtained by considering only the coefficient of the diverging term. The general expression of the electric field can be written as

$$
\begin{aligned}
\vec{E}_{\mathrm{TM}}= & i e^{i \omega t} A \frac{c^{2} \mu a_{g} \omega}{L^{2} \pi a} \\
\times & \sum_{n, v, r} \frac{1}{1-\left(\omega / \omega_{n v r}\right)^{2}} \frac{\varepsilon_{n}}{\omega_{n v r}^{2} x_{n v} J_{n+1}^{2}\left(x_{n v}\right)}\left\{\begin{array}{c}
\operatorname{Re}\left\{I_{n \nu}^{\mathrm{TM}}\right\} \\
\operatorname{Im}\left\{I_{n \nu}^{\mathrm{TM}}\right\}
\end{array}\right\} \\
\times & {\left[\frac{r x_{n v}}{a} J_{n}^{\prime}\left(\frac{x_{n v} \rho}{a}\right) \sin \left(\frac{r \pi z}{L}\right)\left\{\begin{array}{c}
\sin n \phi \\
\cos n \phi
\end{array}\right\} \hat{e}_{\rho}\right.} \\
& +\frac{r n}{\rho} J_{n}\left(\frac{x_{n \nu} \rho}{a}\right) \sin \left(\frac{r \pi z}{L}\right)\left\{\begin{array}{c}
\cos n \phi \\
-\sin n \phi
\end{array}\right\} \hat{e}_{\phi} \\
& \left.-\frac{L x_{n v}^{2}}{\pi a^{2}} J_{n}\left(\frac{x_{n \nu} \rho}{a}\right) \cos \left(\frac{r \pi z}{L}\right)\left\{\begin{array}{c}
\sin n \phi \\
\cos n \phi
\end{array}\right\} \hat{e}_{z}\right],
\end{aligned}
$$




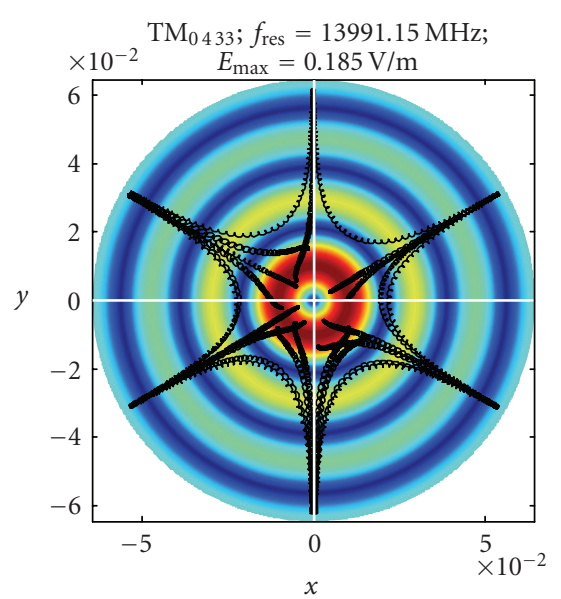

(a)

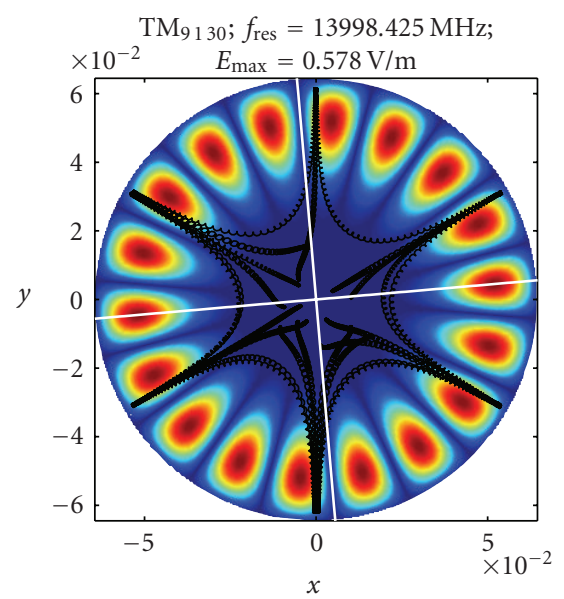

(d)

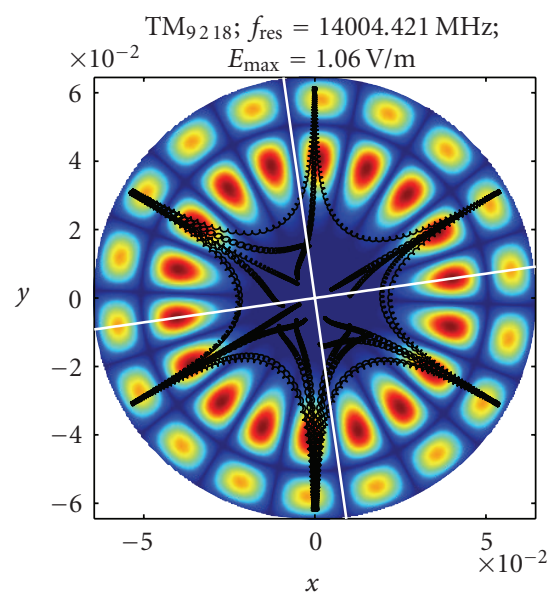

(g)

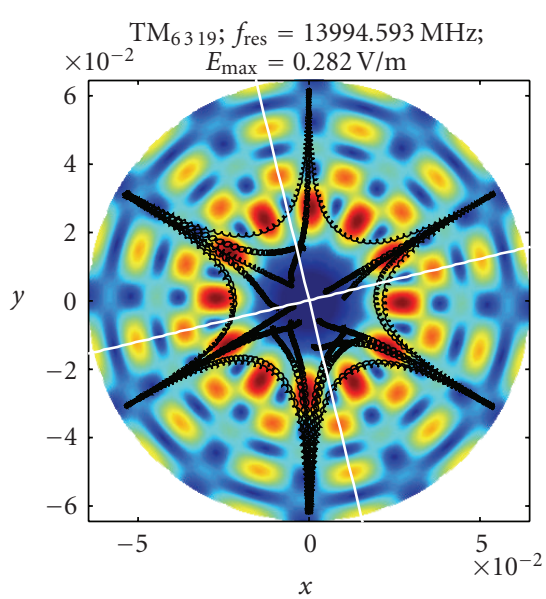

(b)

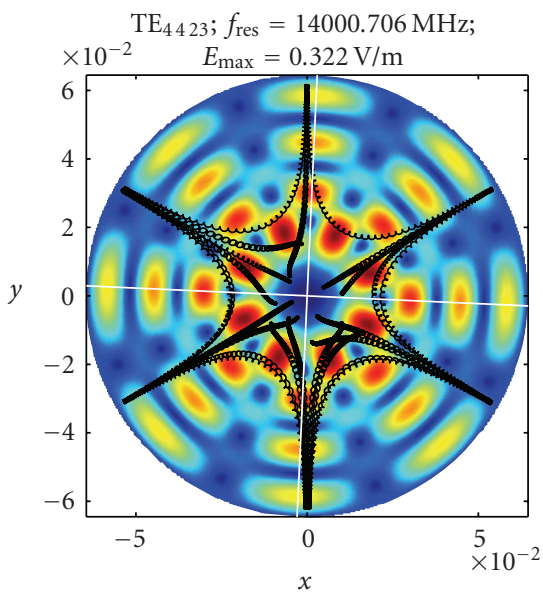

(e)

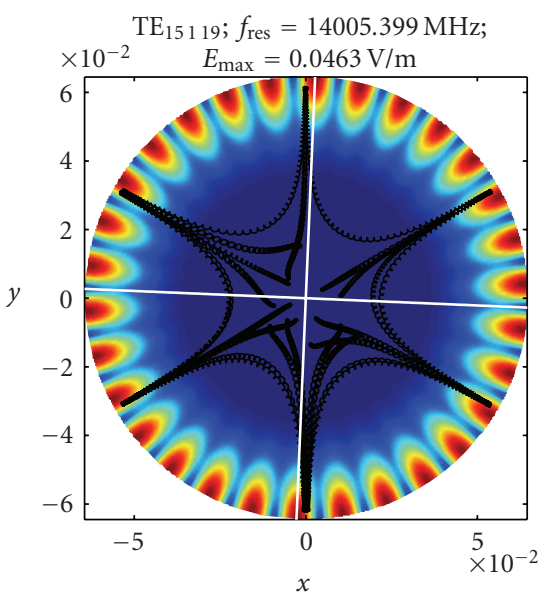

(h)

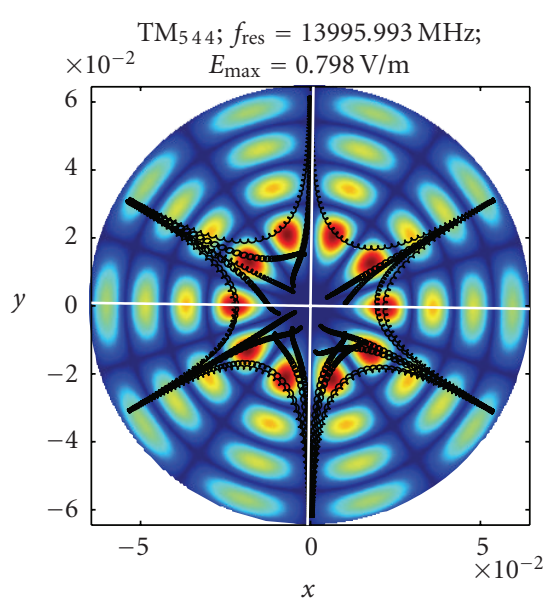

(c)

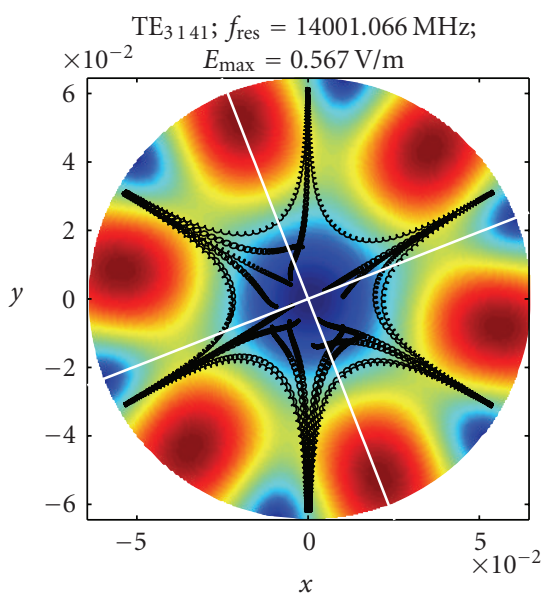

(f)

FIgURE 2: Modulus of the electric field without the term $\left\{1 /\left[1-\left(\left(\omega / \omega_{n v r}\right)^{2}\right]\right\}\right.$ in the plane $z=L / 2$ for modes close to the 14 GHz frequency for $1 \mathrm{~W}$ of the incoming wave power. For each mode the maximum field value is estimated after an equal time interval, supposed large enough to neglect the second-order terms. The white normal axes indicate the mode rotation caused by the coupling with the rectangular waveguide. 
for the TM modes, with $\varepsilon_{n}$ the Neumann's factor (equal to 1 for $n=0$, equal to 2 if $n=1,2, \ldots), x_{n v}$ the $v$-root of the Bessel function of $n$ order, and

$$
\begin{aligned}
I_{n \nu}^{\mathrm{TM}}=-\int_{S} \sin \left[\frac{\pi\left(x-x_{A}\right)}{a_{g}}\right] & {\left[e^{-i(n+1) \phi} J_{n+1}\left(\frac{x_{n \nu} \rho}{a}\right)\right.} \\
& \left.+e^{-i(n-1) \phi} J_{n-1}\left(\frac{x_{n \nu} \rho}{a}\right)\right] d S,
\end{aligned}
$$

and for the TE modes

$$
\begin{aligned}
\vec{E}_{\mathrm{TE}}= & i e^{i \omega t} A \frac{c^{2} \mu a_{g} \omega}{L^{2} \pi a} \\
& \times \sum_{n, v, r} \frac{1}{1-\left(\omega / \omega_{n v r}\right)^{2}} \frac{\varepsilon_{n} r x_{n v}^{\prime}}{\omega_{n v r}^{2}\left(x_{n v}^{\prime 2}-n^{2}\right) J_{n}^{2}\left(x_{n v}^{\prime}\right)} \\
& \times\left\{\begin{array}{l}
\operatorname{Im}\left\{I_{n \nu}^{\mathrm{TE}}\right\} \\
\operatorname{Re}\left\{I_{n \nu}^{\mathrm{TE}}\right\}
\end{array}\right\} \sin \left(\frac{r \pi z}{L}\right) \\
& \cdot\left[\frac{n}{\rho} J_{n}\left(\frac{x_{n v}^{\prime} \rho}{a}\right)\left\{\begin{array}{c}
\cos n \phi \\
-\sin n \phi
\end{array}\right\} \hat{e}_{\rho}\right. \\
& \left.-\frac{x_{n v}^{\prime}}{a} J_{n}^{\prime}\left(\frac{x_{n \nu}^{\prime} \rho}{a}\right)\left\{\begin{array}{c}
\sin n \phi \\
\cos n \phi
\end{array}\right\} \hat{e}_{\phi}\right],
\end{aligned}
$$

with $x_{n v}^{\prime}$ the $v$-root of the first derivative of the Bessel function of $n$ order and

$$
\begin{aligned}
& I_{n \nu}^{\mathrm{TE}}=\int_{S} \sin \left[\frac{\pi\left(x-x_{A}\right)}{a_{g}}\right]\left[-e^{i(n+1) \phi} J_{n+1}\left(\frac{x_{n \nu}^{\prime} \rho}{a}\right)\right. \\
& \left.+e^{i(n-1) \phi} J_{n-1}\left(\frac{x_{n \nu}^{\prime} \rho}{a}\right)\right] d S \text {. }
\end{aligned}
$$

The integrals (6) and (8) represent the coupling for the TM and TE cases, giving a different amount of energy to be transferred from the incoming electromagnetic wave to each mode. In Figure 2, the modulus of the electric field without the term $1 /\left(1-\left(\omega / \omega_{n v r}\right)^{2}\right)$ is shown in the $x y$ plane for $z=$ $L / 2$, for the modes with resonance between $13991.15 \mathrm{MHz}$ and 14005.44 MHz. For each of them, the geometric degeneration is uniquely resolved by the exciting waveguide, and the related rotation is indicated by two normal white axes. The maximum modulus has been calculated for $1 \mathrm{~W}$ of the waveguide power, and after a time interval large enough to neglect the second-order terms. It can be an estimation of the actual energy coupled to each mode.

\section{CONCLUSION}

It has been shown that the field distribution inside the cavity of an electron cyclotron resonance ion source can be changed significantly by means of small frequency variations that have a huge effect both on the spatial distribution of minima and maxima and on the amount of energy coupled from the feeding waveguide to the cavity. The modes have a completely different pattern. Preliminary calculations, not shown here, indicated that some electromagnetic field configurations are more effective to accelerate the confined electrons, because of their proper spatial intensity distribution respect to the star shaped trajectory, representative of the particle motion in presence of the magnetostatic field only. It has a direct effect on the rate of ionisation and therefore on the plasma formation.

\section{REFERENCES}

[1] S. Gammino, "3rd and 4th generation ECRIS: some possible scenarios," in Proceedings of the 17th International Workshop on ECR Ion Sources and Their Applications, IMP, Lanzhou, China, September 2006.

[2] J. Van Bladel, Electromagnetic Fields, Springer, Berlin, Germany, 1985.

[3] S. Gammino and G. Ciavola, "The contribution of the INFNLNS to the development of electron cyclotron resonance ion sources," Review of Scientific Instruments, vol. 71, no. 2, pp. 631636, 2000.

[4] F. Consoli, S. Barbarino, L. Celona, G. Ciavola, S. Gammino, and D. Mascali, "Investigation about the modes in the cylindrical cavity of an ECR ion source," Radiation Effects and Defects in Solids, vol. 160, no. 10-12, pp. 467-475, 2005.

[5] J. C. Slater, Microwave Electronics, Van Nostrand, London, UK, 1963. 

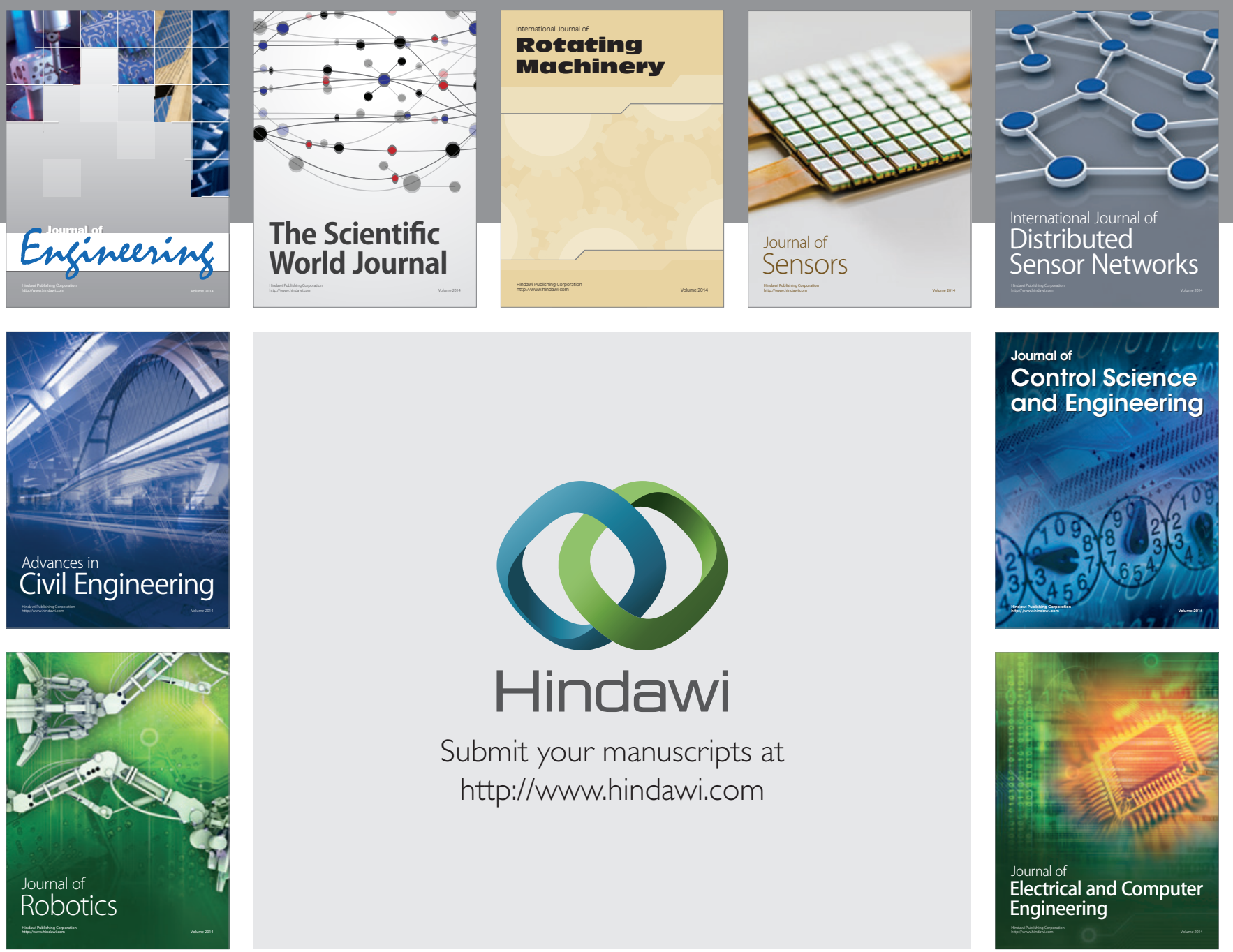

Submit your manuscripts at

http://www.hindawi.com
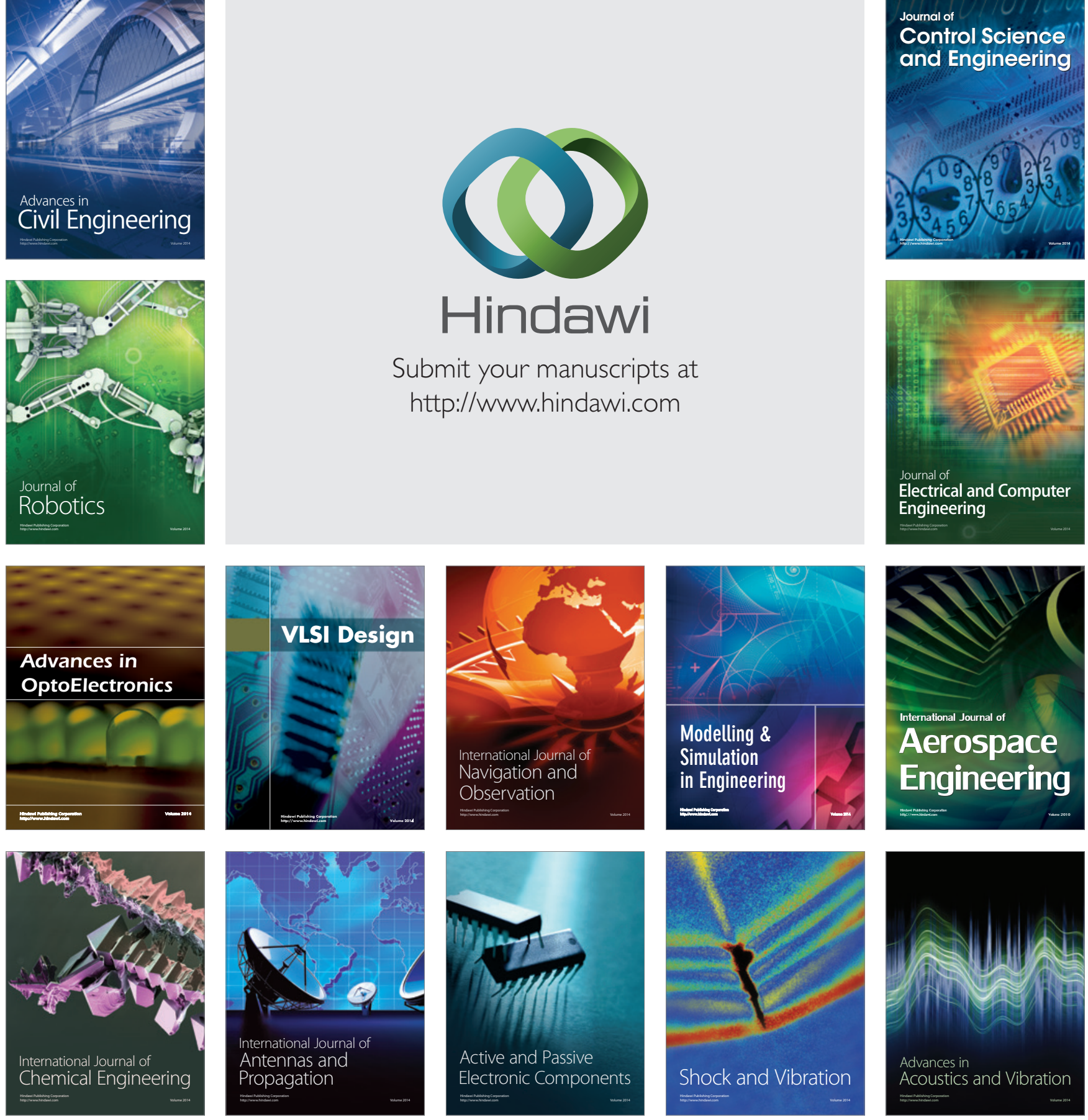